\title{
Interfacial Fracture Toughness Comparison of Three Indirect Resin Composites to Dentin and Polyether Ether Ketone Polymer
}

\author{
Rayhaneh Khalesi ${ }^{1,2}$ Mahdi Abbasi ${ }^{1}$ Zahra Shahidi ${ }^{1}$ Masoumeh Hasani Tabatabaei ${ }^{1}$ Zohreh Moradi ${ }^{1}$ \\ ${ }^{1}$ Department of Restorative Dentistry, Tehran University of Medical \\ Address for correspondence Zohreh Moradi, DDS, MSc, Department \\ of Restorative Dentistry, Dental Faculty, Tehran University of Medical \\ Sciences, North Amirabad Avenue, Box 1439955991, Tehran, Iran \\ 2Department of Restorative Dentistry, Ahwaz Jundishapur \\ University of Medical Sciences, Ahwaz, Iran \\ (e-mail: zohrehmoradi2003@yahoo.com).
}

Eur J Dent:2020;14:456-461

\begin{abstract}
Objectives Advances in laboratory composites and their high wear resistance and fracture toughness have resulted in their growing popularity and increasing use for dental restorations. This study sought to assess the fracture toughness of three indirect composites bonded to dental substrate and polyether ether ketone (PEEK) polymer.

Materials and Methods This in vitro study was conducted on two groups of dental and polymer substrates. Each substrate was bonded to three indirect composite resins. Sixty blocks $(3 \times 3 \times 12 \mathrm{~mm})$ were made of sound bovine anterior teeth and PEEK polymer. Sixty blocks $(3 \times 3 \times 12 \mathrm{~mm})$ were fabricated of CRIOS (Coltene, Germany), high impact polymer composite (HIPC; Bredent, Germany), and GRADIA (Indirect; GC, Japan) composite resins. Composites were bonded to dentin using Panavia F 2.0 (Kuraray, Japan). For bonding to PEEK, Combo.lign (Bredent) and Visio.Link (Bredent) luting cements were used. In all samples, a single-edge notch was created by a no. 11 surgical blade at the interface. The samples were subjected to 3,500 thermal cycles, and their fracture toughness was measured in a universal testing machine (Zwick) Roell, Germany) by application of four-point flexural load.

Statistical Analysis Data were analyzed using one-way analysis of variance, Kruskal-Wallis.

Keywords

- composite resin

- fracture strength

- polyether ether ketone

Results The fracture toughness of CRIOS-PEEK interface was significantly higher than HIPC-PEEK. The fracture toughness of GRADIA-PEEK was not significantly different from that of HIPC and CRIOS. The fracture toughness of GRADIA-dentin was significantly higher than HIPC-dentin.

Conclusion Considering the limitations of this study, GRADIA has the highest bond strength to dentin, while CRIOS shows the highest bond strength to PEEK.
\end{abstract}

\section{Introduction}

High demand for esthetic anterior restorations has resulted in advances in all-ceramic and indirect composite resin restorations for veneering of teeth. Laboratory veneers are believed to be the most esthetic and conservative type of veneering. ${ }^{1,2}$ The use of indirect esthetic restorations has greatly increased in the recent years, which may partly be due to the high failure rate of direct composite restorations ${ }^{3}$ and associated problems such as marginal discoloration, marginal ditching, and wear. ${ }^{4,5}$ Stresses generated in composites following their polymerization shrinkage are also responsible for failure of composite restorations. ${ }^{3,5}$ 
Indirect composite restorations generate lower polymerization shrinkage stresses and have lower rate of the above-mentioned complications. ${ }^{3,5}$ Evidence shows that secondary heating for polymerization of indirect composites during their fabrication process reinforces their physical and mechanical properties. ${ }^{6,7}$

Aside from the advantages of indirect composite restorations, their bonding to tooth structure is still challenging. ${ }^{8}$ The rate of debonding is relatively high due to the degradation of the cement-veneering bonding interface. ${ }^{3}$ Advances in adhesive systems and luting and bonding cements allow obtaining a stable and reliable bonding between the tooth structure and restoration. ${ }^{3,4}$

Bond strength tests do not assess the properties of materials and the results of these tests highly depend on factors such as size of samples, load application tool, inherent properties of materials, and presence of cracks or defects at the interface. ${ }^{9,10}$

Since the existing defects and cracks affect the bond strength, fracture toughness test, which can better concentrate the bonding stresses at the actual interface may be more suitable for assessment of the mechanical properties and efficacy of bonding to dentin and enamel. ${ }^{10,11}$ Dental substrate is often used for assessment of bond strength.

The polyether ether ketone (PEEK) is an aromatic polymer used in orthopaedics and dentistry for its favorable properties such as high flexural strength and hardness. It is used for the fabrication of dental prosthesis, implants, splints, abutments temporary restorations, or clasps of removable dentures. Due to the low translucency of PEEK, we have to paint it or cover it with ceramic or composite veneers for its esthetic application in the oral cavity..$^{12-15}$

Considering the use of PEEK in contemporary dentistry, bonding to both dental substrate and PEEK polymer was evaluated in our study. This study aimed to assess the fracture strength of three indirect composites bonded to dental substrate and PEEK.

\section{Materials and Methods}

\section{Sample Preparation}

This in vitro experimental study was conducted on three indirect composites that were bonded to dental and polymer substrates in six subgroups. - Table 1 shows the materials used in this study.

A total of 30 sound anterior bovine teeth extracted within the past 6 months were collected. The teeth were stored in
$0.5 \%$ chloramine $\mathrm{T}$ solution at $4^{\circ} \mathrm{C}$ after extraction. The teeth were mounted in an acrylic mold. Incisal enamel of the teeth was removed by a cutting saw (Mecatome T; Pressi, France), then they longitudinally sectioned into dentin blocks measuring $3 \times 3 \times 12 \mathrm{~mm}$ for bonding to indirect composites. The bars were carefully inspected under a stereomicroscope to ensure absence of pulp tissue residues, cracks, or defects.

Thirty PEEK polymer blocks were also fabricated in dimensions similar to those of dental substrates for bonding to indirect composites.

Twenty composite samples with square-shaped cross-sections were also fabricated of CRIOS composite and high impact polymer composite (HIPC) and sectioned into blocks measuring $3 \times 3 \times 12 \mathrm{~mm}$ by a Mecatome (201; Pressi, France).

For the fabrication of GRADIA indirect samples, a twopiece steel mold with internal dimensions of $3 \times 3 \times 12 \mathrm{~mm}$ was used. Composite was applied into the mold in six increments each with 2-mm thickness and packed. Each layer was light cured for 40 seconds using a LED light curing unit (Wood Pecker; Qudent, China) with 375 to 570 nm wavelength and a light intensity of $400 \mathrm{~mW} / \mathrm{cm}^{2}$ measured by a radiometer. Then, they were placed in a laboratory light curing unit (Eurolight, Taiwan) for 3 minutes according to the manufacturer's instructions. All composite blocks were inspected under a stereomicroscope (SMZ 745T; Nikon, Japan) to ensure absence of cracks and defects/voids.

To better simulate the clinical setting, Panavia F 2.0 dual cure cement (Kuraray, Japan) was used for bonding of dental substrates to composites. Combo.lign (Bredent, Germany) and Visio.Link (Bredent) luting cements were used for bonding of composite to PEEK according to the manufacturer's instructions.

\section{Preparation of Indirect Composite Samples}

As recommended by the manufacturers of cements used in this study, the entire bonding surface area of laboratory composites was sandblasted with $50 \mu$ aluminum oxide particles with 5 psi ( $0.034 \mathrm{MPa}$ ) pressure, approximate distance of 3 to $5 \mathrm{~mm}$, and approximate angle of 60 to 90 degrees relative to the surface for 10 seconds.

\section{Preparation of Dental Substrate}

Dental substrates (stored in saline) were prepared by highspeed diamond bur (006; Tizkavan, Iran) to create adequate amount of standard smear layer. ${ }^{16}$ The bonding surface area of composite blocks was etched for 5 seconds using K-Etchant (Kuraray) to remove impurities and was then rinsed and

Table 1 Types of indirect composite resins used in this study

\begin{tabular}{|l|l|l|l|}
\hline Material & Manufacturer & Type & Composition \\
\hline Brilliant CRIOS & Coltene & Submicron hybrid & $\begin{array}{l}\mathrm{SiO}_{2} \text {, cross-linked methacrylate, } \\
\text { nonpolymerized resin matrix }\end{array}$ \\
\hline GRADIA & GC & $\begin{array}{l}\text { Hybrid microfilled reinforce } \\
\text { composite }\end{array}$ & $\begin{array}{l}\text { Silica, all silicate glass, prepolymerized filler } \\
\text { with UDMA }\end{array}$ \\
\hline HIPC & Bredent & $\begin{array}{l}\text { Amorphous cross linked polymethyl } \\
\text { methacrylate, microceramic fillers }\end{array}$ \\
\hline
\end{tabular}

Abbreviations: HIPC, high impact polymer composite; UDMA, urethane dimethacrylate. 
dried. Bis-Silane two-component ceramic primer (Bisco, United States) was rubbed on the bonding surface, allowed 30 seconds, and gently air dried for 20 seconds.

Dental substrates were also etched for 10 seconds using K-Etchant (Kuraray) and were then rinsed and air dried to obtain a glossy, moist (not too dry and not too wet) surface. ED Primer II (A + B; Kuraray) was applied on dental substrate bonding surface, allowed 30 seconds, and excess material was removed by a microbrush and gently air sprayed. Next, A and B pastes of Panavia cement were equally mixed and applied on the bonding surface area of composite and dental substrate. The samples were pressed by gentle finger pressure on the smooth surface of a glass slab in order for the excess cement to leak out. Excess cement was removed and the interface was light-cured for 20 seconds from each side using the LED light curing unit (Wood Pecker).

\section{Preparation of PEEK Substrate}

As recommended by the manufacturer, the bonding surface of this substrate was sandblasted with $50 \mu$ aluminum oxide particles with 5 PSI (0.034 MPa) pressure, approximate distance of 3 to $5 \mathrm{~mm}$, and approximate angle of 60 to 90 degrees relative to the surface for 10 seconds using a sandblaster (Constellation Mestra, Spain). All surfaces of the samples, including indirect composites (CRIOS, HIPC, and GRADIA) and PEEK polymer (except for the bonding surface) were covered with adhesive tape to prevent defect in the walls during sandblasting, except for the bonded surfaces. The bonding surface in all three groups of indirect composites and PEEK polymer was cleaned with a gauze dipped in ethylic alcohol for 5 seconds to eliminate impurities. Visio. Link (Bredent) bonding agent was applied on the bonding surface of the indirect composite groups and PEEK polymer substrate such that a glossy surface was obtained. Light curing was then performed using a LED light curing unit (Wood Pecker). Combo.lign dual cure luting cement (Bredent) was then applied on the bonding surface of indirect composite samples and PEEK polymer substrate and the two bonding surface areas were pressed on each other with gentle finger pressure in order for the excess cement to leak out. Excess cement was removed and the interface was light cured for 180 seconds from each side using the LED light curing unit (Wood Pecker).

A single-edge notch was then created at the interface with $0.3 \mathrm{~mm}$ height using a no. 11 scalpel with a diameter less than $0.3 \mathrm{~mm}$ under a stereomicroscope (SMZ 745T; Nikon, Japan). The samples were then subjected to thermocycling for 3,500 cycles between 5 and $55^{\circ} \mathrm{C}$ with a dwell time of 5 seconds and transfer time of 30 seconds to simulate the oral clinical setting. ${ }^{17}$

\section{Measurement of Fracture Toughness}

After thermocycling, the samples were subjected to fracture toughness testing in a universal testing machine by application of four-point flexural load, according to ASTM standard E399-83. The outer and inner spans were 20 and $10 \mathrm{~mm}$, respectively. Load was applied at a crosshead speed of $0.1 \mathrm{~mm} / \mathrm{s}$ until failure. Fracture toughness was calculated using the following formula:

$$
K=P \sqrt{\alpha} / \text { bd }
$$

Where $d=0.3 \mathrm{~mm}, a=$ width of sample in millimeters, and $b=$ thickness of sample in millimeters.

Data were analyzed using one-way analysis of variance (ANOVA), Kruskal-Wallis.

\section{Results}

- Table 2 shows the mean fracture toughness of interface for each composite group.

\section{Fracture Toughness of Composite Samples Bonded to PEEK}

As reported in - Table 3, one-way ANOVA showed a significant difference in fracture toughness of composites bonded to PEEK ( $p=0.01)$. The Tukey's honestly significant difference test showed that the fracture toughness of interface of CRIOS-PEEK was significantly higher than that of HIPC-PEEK $(p=0.00)$. The fracture toughness of GRADIA-PEEK was not significantly different from that of CRIOS and HIPC $(p>0.05)$.

\section{Fracture Toughness of Composite Samples Bonded to Dental Substrate}

The results of the Kruskal-Wallis' test showed that the difference in fracture toughness of composites bonded to dental substrate was statistically significant $(p=0.007)$. The fracture toughness of GRADIA-dentin was significantly higher than that of HIPC-dentin. The fracture toughness of CRIOS group was not significantly different from that of GRADIA and HIPC ( - Fig. 1).

Table 2 Mean fracture toughness of the bonding interfaces (MPa.m $\mathrm{m}^{1 / 2}$ )

\begin{tabular}{|l|l|l|l|l|l|l|}
\hline Substrate & Composite & $N$ & Minimum & Maximum & Mean & $\begin{array}{l}\text { Standard } \\
\text { deviation }\end{array}$ \\
\hline \multirow{3}{*}{ PEEK } & CRIOS & 10 & 0.85 & 2.23 & 1.4270 & 0.47157 \\
\cline { 2 - 8 } & GC & 10 & 0.39 & 2.08 & 1.1150 & 0.43480 \\
\cline { 2 - 8 } & HIPC & 10 & 0.40 & 1.21 & 0.8430 & 0.26226 \\
\hline \multirow{3}{*}{ Dentin } & CRIOS & 10 & 0.00 & 0.41 & 0.1180 & 0.13726 \\
\cline { 2 - 8 } & GC & 10 & 0.00 & 0.87 & 0.3670 & 0.28787 \\
\cline { 2 - 8 } & HIPC & 10 & 0.00 & 0.18 & 0.0290 & 0.05763 \\
\hline
\end{tabular}


Table 3 Comparison of composite-PEEK groups using one-way ANOVA test

\begin{tabular}{|l|l|l|l|l|l|}
\hline & Sum of squares & Difference & Mean square & $F$ & Significance \\
\hline Between groups & 1.708 & 2 & 0.854 & 5.335 & 0.011 \\
\hline Within groups & 4.322 & 27 & 0.160 & & \\
\hline Total & 6.030 & 29 & & & \\
\hline
\end{tabular}

Abbreviations: ANOVA, analysis of variance; PEEK, polyether ether ketone. Note: The mean difference is significant at the 0.05 level.

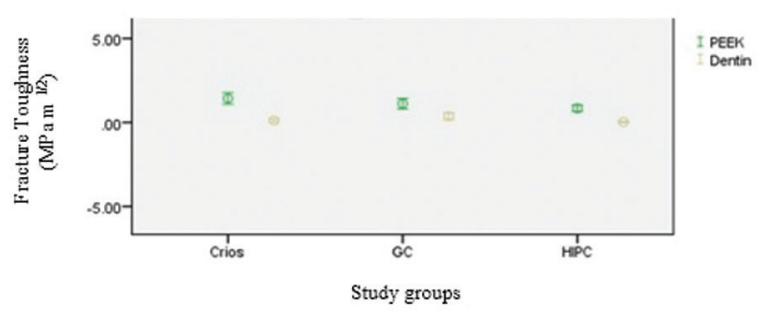

Fig. 1 Mean interfacial fracture toughness values of the study groups with $95 \%$ confidence interval. PEEK, polyether ether ketone.

\section{Discussion}

In this study, the bond strength of three types of indirect composites to dentin and PEEK substrates was compared by analyzing the fracture toughness of the bonding interface. The results showed that the bond strength of GRADIA to dentin was higher than the other composites and the highest bond strength for PEEK substrate was observed for CRIOS composite. HIPC composite showed the lowest bond strength to both substrates.

At present, esthetics plays an important role in dental treatment planning. ${ }^{4}$ Although amalgam and gold restorations yield long-term favorable results, they are not often accepted by patients. ${ }^{16}$ Laboratory composites used in the form of inlays, onlays, and prefabricated veneers have optimal wear resistance especially in contact with the opposing teeth. They have lower polymerization shrinkage, which is limited to the thin layer of luting cement in contact with the tooth structure. Thus, they are suitable for restoration of large defects..$^{18,19}$

Considering the small size of human teeth and since bovine teeth are very similar to human teeth in terms of morphology and structure, we used bovine teeth as dental substrate in this study since they are larger than human teeth. ${ }^{20,21}$

It appears that many of the recorded bond strength values do not correctly represent the highest stress that can be tolerated by adhesive prior to failure because the bond strength is often calculated by measuring the maximum shear or tensile load at failure divided by the bonding surface area. This approach is based on complete stress distribution at the interface during failure. However, it does not often occur in reality due to differences in modulus of elasticity and Poisson's ratio of different materials. These differences cause unequal transverse contractions at the adhesive interface and create complex stresses at this area. Stress analyses have shown highest stress accumulation at the periphery and circumference of the adhesive layer. Moreover, defects tend to appear in this high-stress area especially in sectioned or machined samples. Thus, cracks often initiate at this area and propagate toward the center of samples. Considering the mechanism of fracture in dentistry, fracture toughness was measured in this study to assess the bond strength of composite to tooth structure. ${ }^{9-11}$

De Munck et al compared the bond strength measured by fracture toughness test and microtensile bond strength test. They used modified chevron notch beam ISO 24370 and measured the bond strength of six types of adhesives to tooth structure. They showed that fracture toughness and microtensile bond strength had a strong correlation. ${ }^{10}$

Several studies have assessed the bond strength at the interface of indirect composites and different substrates by measuring fracture toughness by single-edge notch method according to ASTM standard E399-83.,2,22,23 In our study, considering the limitations such as small size of dental substrates, the single-edge notch method based on ASTM standard E399-83 was applied.

GRADIA contains reactive groups because it is not fabricated in blocks under pressure and heat and its polymerization is done in Labolight device, and therefore, is not complete. Thus, it still contains molecules with double bonds, which can form chemical bonds with Panavia adhesive cement molecules, which contain 10-methacryloyloxydecyl dihydrogen phosphate (10-MDP).Polymerization rate is $99 \%$ in HIPC and thus, no molecule remains for bond with Panavia molecules or they are scarce in number. CRIOS composite contains $\mathrm{NH} / \mathrm{OH}$, which can form hydrogen bonds with Panavia molecules. This composite can also chemically bond to MDP molecules of silane. Also, it forms strong chemical bonds with cement molecules during the process of interloping. This explains stronger bond of CRIOS and weaker bond of HIPC with Visio.Link molecules to PEEK. In 2003, El Zohairy et al assessed the microtensile bond strength between luting cements and ceramic and composite blocks prior to different surface treatments and reported that the bond strength between cement and composite blocks was higher than that of cement and ceramics, which was due to the mechanical properties of materials and their structural content. ${ }^{3}$ In our study, mechanical surface roughening was performed by sandblasting with aluminum oxide particles as recommended by the 
manufacturer and then silane was applied for mechanical and chemical bonding between the composite and luting cement.

Fuentes et al in 2013 reported a correlation between application of silane and bond strength of different cements to indirect composite inlays. ${ }^{24}$ In our study, the presence of silane in the cementation protocol of CRIOS and GRADIA laboratory composites resulted in their stronger bond to MDP molecules in Panavia cement and this was due to the presence of functional molecules in nonpolymerized resin of these two composites. Therefore, lower bond strength to HIPC is due to the lower presence of functional molecules in its polymer-saturated resin.

PEEK is a thermoplastic semicrystalline polymer. It has optimal properties that make it suitable for use in restorative dentistry and prosthodontics. Despite optimal biological and mechanical properties, PEEK cannot be used alone in esthetic regions due to its unaesthetic appearance. ${ }^{12-15}$ Therefore, one objective of this study was to find an indirect composite with optimal mechanical properties and bond strength for veneering and masking the color of PEEK. Our results showed that HIPC yielded the lowest bond strength to PEEK compared with the other two composites. The same result was found for bonding to dental substrate, which is due to the absence of active molecules with double bonds to form a strong bond with Combo.lign cement at the interface. Among composite groups, CRIOS-PEEK group showed the highest bond strength, while among dental substrate groups, GRADIA showed significantly higher bond strength. This difference may be due to the use of different cements in the two groups of polymer and dental substrates (Panavia F 2.0 and Combo.lign).

Different surface conditioning such as acid etching, sandblasting, plasma or laser techniques, as well as different primers and adhesives has been used to establish optimal bonding to PEEK. Stawarczyk et al in 2014 assessed the tensile bond strength of a veneering composite and PEEK following different surface preparations and reported that sandblasting with aluminum oxide particles caused a greater increase in shear bond strength than other treatments. ${ }^{15}$ In 2013 , Stawarczyk et al found that air abrasion followed by application of Visio. Link and Signum PEEK Bond significantly improved the tensile bond strength between PEEK and veneering resins, before and after thermocycling. ${ }^{25}$ Caglar et al reported that using adhesive systems, including Visio.Link after sandblasting improved resin bonding to PEEK. ${ }^{26}$ In the current study, the surface of indirect composites and PEEK were sandblasted with aluminum oxide particles according to the manufacturers' instructions to increase the bond strength. We also used Visio.Link bonding agent to achieve optimal bond strength between different veneering resins and PEEK.

\section{Conclusion}

Considering the limitations of this study, GRADIA has the highest bond strength to dentin, while CRIOS shows the highest bond strength to PEEK. The bond strength of HIPC to dentin and PEEK was significantly lower than that of the other two composites.

\section{Funding}

This study was part of a M.S. thesis supported by Tehran University of Medical Science (TUMS); Grant no. 95-04-69-33541.

\section{Conflict of Interest}

None declared.

\section{Acknowledgments}

This study was derived from a thesis for a master's degree in restorative dentistry. The authors would like to acknowledge Dr. Mohammad Javad Kharazi Fard Statistical Advisor, Dental Research Center, Tehran University of Medical Science, Tehran, Iran for preforming statistical analysis.

\section{References}

1 Sano $\mathrm{H}$, Shono T, Sonoda H, et al. Relationship between surface area for adhesion and tensile bond strength-evaluation of a micro-tensile bond test. Dent Mater 1994;10(4):236-240

2 Tam LE, McComb D. Shear bond strengths of resin luting cements to laboratory-made composite resin veneers. J Prosthet Dent 1991;66(3):314-321

3 El Zohairy AA, De Gee AJ, Mohsen MM, Feilzer AJ. Microtensile bond strength testing of luting cements to prefabricated CAD/CAM ceramic and composite blocks. Dent Mater 2003;19(7):575-583

4 Perdigão J, Sezinando A, Muñoz MA, Luque-Martinez IV, Loguercio AD. Prefabricated veneers - bond strengths and ultramorphologicalanalyses.JAdhes Dent 2014;16(2):137-146

5 Cetin AR, Unlu N, Cobanoglu N. A five-year clinical evaluation of direct nanofilled and indirect composite resin restorations in posterior teeth. Oper Dent 2013;38(2):E1-E11

6 Wendt SL Jr. The effect of heat used as secondary cure upon the physical properties of three composite resins. II. Wear, hardness, and color stability. Quintessence Int 1987;18(5):351-356

7 Nguyen JF, Migonney V, Ruse ND, Sadoun M. Resin composite blocks via high-pressure high-temperature polymerization. Dent Mater 2012;28(5):529-534

8 Zorba YO, Ilday NO, Bayındır YZ, Demirbuga S. Comparing the shear bond strength of direct and indirect composite inlays in relation to different surface conditioning and curing techniques. Eur J Dent 2013;7(4):436-441

9 De Munck J, Poitevin A, Lührs AK, et al. Interfacial fracture toughness of aged adhesive-dentin interfaces. Dent Mater 2015;31(4):462-472

10 De Munck J, Luehrs AK, Poitevin A, Van Ende A, Van Meerbeek B. Fracture toughness versus micro-tensile bond strength testing of adhesive-dentin interfaces. Dent Mater 2013;29(6):635-644

11 Moharamzadeh K, Hooshmand T, Keshvad A. Van Noort R. Fracture toughness of a ceramic-resin interface. Dent Mater 2008;24(2):172-177

12 Kern M, Lehmann F. Influence of surface conditioning on bonding to polyetheretherketon (PEEK) Dent Mater 2012;28(12):1280-1283

13 Rosentritt M, Preis V, Behr M, Sereno N, Kolbeck C. Shear bond strength between veneering composite and PEEK after different surface modifications. Clin Oral Investig 2015;19(3): 739-744 
14 Taufall S, Eichberger M, Schmidlin PR, Stawarczyk B. Fracture load and failure types of different veneered polyetheretherketone fixed dental prostheses. Clin Oral Investig 2016;20(9):2493-2500

15 Stawarczyk B, Jordan P, Schmidlin PR, et al. PEEK surface treatment effects on tensile bond strength to veneering resins. J Prosthet Dent 2014;112(5):1278-1288

16 Barabanti N, Gagliani M, Roulet JF, Testori T, Özcan M, Cerutti A. Marginal quality of posterior microhybrid resin composite restorations applied using two polymerisation protocols: 5-year randomised split mouth trial. J Dent 2013;41(5):436-442

17 Gale MS, Darvell BW. Thermal cycling procedures for laboratory testing of dental restorations. J Dent 1999;27(2):89-99

18 Scheibenbogen-Fuchsbrunner A, Manhart J, Kremers L, Kunzelmann KH, Hickel R. Two-year clinical evaluation of direct and indirect composite restorations in posterior teeth. J Prosthet Dent 1999;82(4):391-397

19 Barabanti N, Preti A, Vano M, Derchi G, Mangani F, Cerutti A. Indirect composite restorations luted with two different procedures: a ten years follow up clinical trial. J Clin Exp Dent 2015;7(1):e54-e59
20 Nakamichi I, Iwaku M, Fusayama T. Bovine teeth as possible substitutes in the adhesion test. J Dent Res 1983;62(10):1076-1081

21 Schilke R, Lisson JA, Bauss O, Geurtsen W. Comparison of the number and diameter of dentinal tubules in human and bovine dentine by scanning electron microscopic investigation. Arch Oral Biol 2000;45(5):355-361

22 Lin L, Drummond JL. Cyclic loading of notched dental composite specimens. Dent Mater 2010;26(3):207-214

23 Ornaghi BP, Meier MM, Lohbauer U, Braga RR. Fracture toughness and cyclic fatigue resistance of resin composites with different filler size distributions. Dent Mater 2014;30(7):742-751

24 Fuentes MV, Ceballos L, González-López S. Bond strength of self-adhesive resin cements to different treated indirect composites. Clin Oral Investig 2013;17(3):717-724

25 Stawarczyk B, Keul C, Beuer F, Roos M, Schmidlin PR. Tensile bond strength of veneering resins to PEEK: impact of different adhesives. Dent Mater J 2013;32(3):441-448

26 Caglar I, Ates SM, Yesil Duymus Z. An in vitro evaluation of the effect of various adhesives and surface treatments on bond strength of resin cement to polyetheretherketone. J Prosthodont 2019;28(1):e342-e349 